Wie die Úbersicht zeigt, kann man also z. B. die Linksäpfolsäure in die Rechtsäpfelsäure verwandeln, indem man sie zunächst durch Phosphorpentachlorid in die Rechtschlorbernsteinsäure und diese unter Benutzung von Silberoxyd in die Rechtsäpfelsäure überführt. Razemisierung tritt bei Anwendung der beiden genannten Hydroxylierungsmittel nur in sehr geringem MaBe, bei Anwendung anderer Hydroxylierungsmittel meist in höherem Grade ein.

Eine Deutung dieser merkwürdigen und lango Zeit ganz unverständlichen Erscheinungen scheint neuerdings durch tberlegungen gefunden zu sein, die etwa gleichzeitig und unabhängig voneinander A. Werner in Zürich und Emil Fischer in Berlin angestellt haben. Die beiden genannten Autoren machen die sehr plausible und in anderen Fällen auch schon als richtig erwiesene Annahme, daB der eigentlichen Substitution eine Addition vorangehe; die eigentliche Substitution würde also erst beim Zerfall des primär gebildeten Additionsproduktes erfolgen. Bei diesem Zerfall braucht nun, das ist das Wesentliche, der neu in das Molekül eintretende Substituent keineswegs die Stelle zu besetzen, die der ausgetretene Substituent innegehabt hat, es können vielmehr bei dem Vorgange konfigurative Verschiebungen eintreten, die im einzelnen vou Fall zu Fall verschieden sein und sicherlich in erheblichem Maße auch von dex Struktur des sich zersetzenden Additionsproduktes abhängen werden. So erscheint das anfangs so rätselhafte Phänomen der Waldenschen Umkehrung wenigstens im Prinzip verständlich, aber es wird noch mancher Arbeit bcdürfon. um zu zeigen, daß diese mögliche Erklärung tatsächlich auch die richtige ist.

\section{Der heutige Stand der Wasserreinigung und Abwässerbeseitigung.}

\section{Von Dr. Hartwig Klut, Berlin,}

Mitglied der Königl. Prüfungsanstalt für Wasserversorgung und Abwässerbeseitigung.

Auf dem für die allgemeine Gesundheitspflege so wichtigen Gebiete der Wasserversorgung und Abwässerbeseitigung der Gemeinden sind in den letzten Jahren nicht unerhebliche Fortschritte zu verzeichnen. Um anch weitero Kreise in den heutigen Stand der Wasserreinigungs- und $\mathrm{Ab}$ wässerboseitigungsfrage einzufuhren, soll im nachstehenden ein kurzer orientierender Utberblick über diese boiden Kapitel der modernon Hygiene gegeben werden. Als Unterlage diento mir nehen anderen Werken das kürzlich aus berufener Feder erschienene, sehr empfehlenswerte Buch von Dr. J. Tillmans: Wasserreinigung und Abuässerbeseitigung, Verlag von Wilholm Knapp, Halle a. S., 1912.

Wasserreinigung.

Es sei znerst die Reiniuung des Wasstrs fur Trinkzueche besprochen.

Durch die klassischen Arbeiten von Robert Koch wissen wir jetzt, daB durch Wasser in erster Linie Trphus and Cholera übcrtragen werden könuen. Es mub daher von cinem zu Trink- und Wirtsehaftszwecken dienenden Wasser gefordert werden, daß dieses vor allen Jingen keine Krankheitserreger ${ }^{1}$ ) aufweist. Des weiteren mub verlangt werden, daß das Wasser keine für Menschen und Tiere sehädliche Bestandteile, z. B. Bleiverbindungen, enthält, und ferner muB es von solcher Beschaffenheit sein, daß es auch zum Genusse ${ }^{2}$ ) anregt.

Zur Entfernung der Bakterien und der Krankheitserreger im Wasser benutzt man im großen die verschiedenen Verfahren der Filtration ${ }^{3}$ ). Als solche dienen:

a) Die langsame oder englische Sandfiltration, wie sie z. B. in Hamburg und Altona angewandt wird. Die Filter stellen im allgemeinen grolie, meist viereckige, von einer Mauer umgebene Flächen dar, welche mit Kies und Sand gefüllt sind. Zu unterst liegt eine Schicht von Steinen von 60 bis $150 \mathrm{~mm}$ Korngröße. Darauf folgt eine Kiesschicht, welche bezweckt, den darüber liegenden Sand zu schützen und die von oben nach unten allmählich größer wird. Die Kiesschicht setzt sich in der Regel zusammen aus einer Lage von etwa NuBstärko, einer von BohnengröBe, einer von Erbsengröße und einer Lage von Hirsekorngrößc (3-5 $\mathrm{mm}$ ). Darauf ruht die Sandschicht, auf welcher das Rohwasser in einer gewissen Höho anfgebracht wird. Sand- und Kiesschicht werden häufig in verschiedener Größe zusammengestollt. Mittlere Stärken sind: $60 \mathrm{~cm}$ Wasserhöhe, $60 \mathrm{~cm}$ Sand und $60 \mathrm{~cm}$ Kies. Das filtrierte Wasser fliekt unten ab und gelangt vou dort in den Reinwasserbehülter. Für die Versorgung einer Stadt mit filtriertem Wasser gebraucht man eine ganze Reihe soleher Sandfilter. Die Filtrationsgeschwindjokeit soll im allgemeinen $0,1 \mathrm{~m}$ in der Stunde nicht überschreiten, eino Geschwindigkeit, boi der also jedes Quadratmeter Filterfläche in der Stunde 100 Liter liefert. Wenn auch durch die Sandfittration kein absoluter Sulutz gegen etwaige im Rohwasser vorhandene Krankheitscrreger erreicht wird, so halt man doch allgemein den relativen Schutz bei geregeltem Betrieb der Filter fïr ziemlich hoch. Zur weitergehenden Reinigung des Wassers wondet man auch, wie z. B. in Bremen, nach E. Götze die Doppelfiltration an. In Mngleburg benutzt man als Vorfiltration die Puëch-Chabal-Filter, sog. Stufenfilter, wodurch die Foinfilter entlastet worden.

b) Schnellfilter. Die Filtrationsgeschwindigkoit lann bei Anwendung von Schnellfiltorn ${ }^{4}$ ) bis zu

1) C. Ginnther, Einfuhrung in das Studium der Bakteriologie, 6. Aufl. Leipzig 1906.

2) R. Abel, Die Vorsebriften zur Sicherung resund heitsgemëßer Trink- und Nutzwasserversorgung. Berlin 1911.

3) O. Spitta, Die Wasserversorgung im IIandbuch dor Gygitue von $M . R a b n c y, M . v$. Gruber U. M. Hicher. II. Band. 2. Abteilung. "Wasser und Abwasser". Jeipzig 1911.

4) E. Friedberger, Versuche über die Verwendbarkeit der amerikanischen Sehnellfiltration für die Königsberger Wasserversorgung, Zeitschr. f. Ilygiene 1908 . Bil. 61, S. 355 . 
$5 \mathrm{~m}$ und mehr in der Stunde gesteigert werden. Als Material benutzt man meist Kies mit einer Korngrösse von 1 bis $3 \mathrm{~mm}$. Zur Erzeugung einer künstlichen Filterdecke dient fast ausschließlich schwefelsaure Tonerde.

Bei Mangel an Trinkwasser greift man zur kïnstlichen Herstellung von Grundwasser aus Oberflächenwasser (Infiltration). Praktische Anwendung macht man hiervon $z$. B. bei den Wasserwerken an der Ruhr. Die sogenannte Uferfiltration besteht darin, daB man am Ufer eines Sees oder FluBlaufes Brunnen absenkt. Aus diesem Brunnen wird dann das Wasser abgepumpt, wodurch der Wasserstand in dem Brunnen erheblich abgesenkt wird. Die Folge davon ist, daß das Wasser aus dem See oder Fluß durch die filtrierenden Sand- oder Kiesschichten des Ufers hindurch in den Brunnen eintritt. Bei diesem Durchgang durch den Boden wird das Wasser in ähnlicher Weise von den Keimen befreit, wie das Grundwasser (z. B. in Offenbach am Main). Für die Gewinnung künstlichen Grundwassers hat C. Reichle ${ }^{1}$ ) folgende Grundsätze aufgestellt:

1. Fehlt für ein Versorgungsgebiet die Möglichkeit einer ausreichenden Versorgung mit Grundwasser, so empfiehlt es sich, bei der Verwendung von Oberflächenwasser in jedem Fall auch die Möglichkeit einer eventuellen kïnstlichen Grundwassererzeugung zu prüfen.

2. Die letztere kann bei sachgemäBer Anlage eine gröBere Betriebssicherheit und einen günstigeren bakteriologischen Effekt als gewöhnliche Sandfilter gewähren; ein wesentJicher Vorteil den letzeren gegenüber bietet aber die Möglichkeit der Erzielung einer gleichmäbigen Temperatur des Wassers.

3. Der Wahl bestimmter Schichten für Anreicherungszwecke hat eine sorgfältige Untersuchung derselben, ihres Aufbaues, ihrer $\mathrm{Zu}$ sammensetzung und ihres Gehaltes an auslaugbaren Stoffen voranzugehen.

4. Es ist notwendig, daB die Bemessung und Anordnung natürlicher Bodenfilter auf Grund sorgfältiger Vorversuche erfolgt, die dabei gewählten Geschwindigkeiten und Wasserspiegelhöhenlagen dürfen im Betrieb nicht ohne weiteres überschritten werden. Bei der Feststellung des Filtrationseffektes ist mit einer zeitweiligen Störung der Filterhaut bzw. der Eintrittsfilterzone überhaupt zu rechnen. Die Aufenthaltsdauer bzw. der Weg des infiltrierten Wassers von der Versickerungsstelle bis zur Fassungsanlage ist so groß zu bemessen, daB auch bei nicht oder unrollständig eingearbeiteten Filterzonen jederzeit eine gesicherte bakteriologische Reinigung erfolgt.

5. Für die Vorreinigung des $z u$ verwendenden Oberflächenwassers von den ihm etwa anhaftenden Verschmutzungen dürften sich ins-

1) C. Reichle, ther kinstliches Grundwasser. Journal f. Casbelenelutung and Wasserversorgung 1010, Bd. 53, S. 699 . besondere grobe Aufstaubecken oder Schnellfilteranlagen empfehlen.

6. Die Anreicherung braucht nicht. nur auf Grundwasserträger aus Sand und Kiesschichten beschränkt zu werden; in bestimmten Fallen dürfen auch geeignete Gesteinsschichten eine künstliche Anreicherung zulassen (künstliches Quellwasser).

7. Anreicherungsanlagen bedürfen wie alle Verfahren zur Reinigung von Oberflächenwasser einer fortlaufenden wissenschaftlichen Kontrolle.

Verfahren der Wassersterilisation. Von den neueren Verfahren zur sicheren Abtötung etwaiger Krankheitserreger im Wasser haben sich im groben bislang bewährt die Ozonisierung ${ }^{1}$ des Wassers, wie sie z. B, in Paderborn geabt wird. Ferner das Ferrochlor-Verfahren Duyk $k^{2}$ ), System Howatson, das mit Erfolg in Middelkerke in Belgien angewandt wird. Das Chlorkalkverfahren, das namentlich in Nordamerika ${ }^{3}$ viel angewandt und auch ganz neuerdings mit Erfolg von einigen Wasserwerken an der $\mathrm{Ruhr}^{4}$ ) benutzt wird. Auch mit Hilfe ultravioletter Strahlen ${ }^{5}$ gelingt es, pathogene Keime zu vernichten. Das Verfahren ist jedoch ziemlich teuer.

Zur Desinfeltion von Wasserleitungen usw. benutzt man mit Erfolg Schwefelsäure (wie z. B. in Beuthen); um Bohrlöcher zu desinfizieren, dient vielfach Karbol-Schwefelsäure ${ }^{6}$ ).

Beschaffenheit des Wassers für eine Zentralversorgung in chemischer Hinsicht. Ein für die Zentralversorgung eines Ortes dienendes Wasser mul auch vom chemischen Standpunkt so beschaffen sein, daB es einmal zum Genusse anregt, and zweitens im Leitungsnetz keine Störungen. wie Zerfressungen oder Verschlammungen des Rchrmaterials. hervorruft. In erster Linie wirkt cin hoher Eisengehalt des Wassers störend. Die reisten Grundwässer der norddeutschen Tiefebene sind mehr oder weniger eisenhaltig. Frisch entnommen sehen solche Wässer in der Regel farblos aus. Durch den Luftzutritt wird das in ilnen enthaltene Eisen oxydiert und ausgeschieden. Hierbei spaltet sich oft Schwefelwasserstoff $a b$. lias Wasser wird trübe und unansehnlich und

1) O. Daslee, Die Reinigung des Trinkwassers durch Ozon, Deutsche Vierteljahrsschrift f. öffentl. Gesundheitspflege 1909 , Bd. 41, $\$$. 385.

2) K. Thumm u. A. Schicle, Mitteilungen aus der Königl. Prufungsanstalt $t$. Wasserversorg. u. $\Lambda$ bwasserbeseitigung, Heft 8 . Berlin 1907. S. 1.

3) K. Imhoff u. Oh, Saville, Die Desinfektion vou Trinkwasser mit Chlorkalk in Nordamerika, Journal f. Gasbeleuchtung u. Wasserversorgung 1910, Bd. 53, S. 1119 .

4) H. Bruns-Gelsenkirchen, Uber die Desinfektion des Trinkwassers in Wasserleitumgen durch Chlorkalk, Journal $i$. Casbeleuchtang n. Wasserversorgung 1912, Bd. 55, S. 640 .

5) Grimm und $R$. Weldert, Sterilisation von Wasser mittels ultravioletter Strahlen, Mitteilungen a. d. Königl. Prüfungsanstalt f. Wasserversorg. $u$, Abwässerbeseitig. Berlin 1911. Heft 14, \$. 85. 1009 . 
scheidet nach einiger Zeit Eisenocker') ab. Ein weiterer Nachteil ist der, daf solche Wässer einen günstigen Nährboden für Eisenbakterien abgeben, die durch ilsr üppiges Wachstum zur Verschlammung der Leitungen und zu den dadurch bedingten Betriebsstörungen noch besonders beitragen. Schon ein Eisengehalt von $0,3 \mathrm{mg}$ im Liter Eisen $\left(\mathrm{Fe}_{2} \mathrm{O}_{3}\right)$ kann ein Leitungsnetz nachteilig beeinflussen.

Je nach der Art der leichteren oder schwereren Ausscheidung des Eisens ${ }^{2}$ ) aus dem Wasser werden die verschiedenen Verfahren der Enteiseming angewendet, die im wesentlichen auf folgewden Vorgängen beruhen:

1. Belüftung und Filtration,

2. Belüftung, Rieselung und Filtration,

3. Benutzung von Chemikalien, wie Alaun, Kalkwasser.

Man kann durch diese Methoden den Eisengehalt im Reinwasser bis unter $0,1 \mathrm{mg}$ im Liter herunterdrücken.

Ahnlich wie das Eisen verhält sich das Mangan im Wasser, das namentlich durch sein Vorkommen im Breslauer und Stettiner Grundwasser erhöhtes Interesse gewonnen hat. $\mathrm{Es}_{\mathrm{s}}$ ist im allgemeinen schwerer áls Eisen aus Wasser ${ }^{3}$ ) zu entfernen, es begünstigt ebenfalls das Wachstum von Manganbakterien. Die Entfernung geschieht im wesentlichen nach den gleichen Grundsätzen wie beim Eisen ${ }^{4}$ ). In gesundhejtlicher Beziehung haben Eisen- und Manganverbindungeu in den Mengen, wie sie im Trinkwasser vorzukommen pflegen, keine Bedientung.

Entfernung der freien Kohlensäure. Wässer, die freie Kohlensäure in größerer Menge gelöst cnthalten, besitzen die nachteilige Eigenschaft, Metalle und Mörtelmaterial anzugreifen. In gesundheitlicher Hinsicht kommt in erster Linie das $B l e i^{5}$ ) in Betracht, das noch vielfach zu Hausanschlüssen rerwendet wird. Selbst sehr geringe Mengen ron Blei im Wasser können schon ernste Erkrankungen bei längerem GenuB solch bleihaltigen Wassers hervorrufen, wie namentlich die Erfahrungen in Dessau es gezeigt haben. Von 28000 Einwohnern litten hier im Jahre 1886 nicht weniger als 92 unter heftigen Vergiftungserscheinungen. Außer diesen schweren Fallen sind naturlich auch sehr viele leichtere Erkrankungen vorgekommen.

Zur Unschädlichmachung dex freien Kohlensäure im Wasser wendet man in der Praxis meist folgende Verfahren an:

1) Vergl. u. a. H. Klut, Gesundheit 1909, Bd. 34, s. 746 .

2) Finger, Wasserversorgung in den Marschen, Klinisches Jahrbuch 1908, Bd. 19.

3) H. Tóbben, Ưber die besten Verfahren der Reinigung des Grundwassers von Eisen und Mangan, Gesundheits-Ingenieur $1907 \mathrm{Nr} .46$ und $4 \%$.

4) Vergl. a. Thiesing, Versuche uber die Entmanganung von Grundwasser (in Stettin), Mitteilg. a. d. Königl. Prufungsanstalt f. Wasservers. u. Abwässerbeseit. Heft 16. Berlin 1912. S. 210.

5) H. Klutt, Bleiröhren und Trinkwasser, Journal f. Gasbeleucht, u. Wasserversorg. 1911, Bd. 54, Nr. 1\%, S. 409 .
Bei weichen Wässern bindet man die freie Kohlensäure durch Filtration des Wassers über Marroor. In Frankfurt am Main hat sich dieses Verfahren nach $J$. Tillmans gut bewährt. Oder man setzt eine berechnete Menge von Sodalösung oder Natronlauge dem Wasser, wie z. B. in Dessau, hinzu. Auch Kíalkwasser wird mit Frfolg, z. B. in WejBwasser (Jausitz), benutzt.

Bei Wässern mit großerer vorübergehender Häle (Karbonathärte) lält sich die freie Kohlensäure ${ }^{1}$ ) durch Rieselung gleichzeitig bei der Enteisenung des Wassers entfornen. H. Wehner') entfernt die freie Kohlensäure aus Wasser durth Rieseling in Takumapraraten.

Reinigung von Trinkuasser im kleinen. Für die Entfernung etwaiger krankheitsverdächtiger Keime in Trinkwasser sind eine Reihe von Kleinund Hausfittern im Gebrauch; sie bestehen meist aus Stein, Kohle, Asbest, Ton, Porzellanerde, Kieselgur usw. Nach O. Spitta ${ }^{3}$ ) dürften die Berkefeld-Filterberzen den relativ besten Kleinfiltertyp darstellen. Sie werden auch in den großen und kleinen Armeefiltern verwendet und sind hier zu einem System von Kerzenbatterien vereinigt. Vergl. a. $K$. Opitz, Brunnenhygiene, Berlin 1910.

Das sicherste Mittel zur Vernichtung von Krankheitserregern im Wasser ist unstreitig das Atikochen. Fine Reihe geeigneter Apparate hierzu beschreibt $J$. Tillmans in seinem obigen Werls an der Hand instruktiver Abbildungen.

Auch im kleinen lasson sich durch chemischphysikalische Behandlung des Wassers mit Ozon und ultrwioletten Strahlen die schädlichen Bakterien aus Wasser mittels zweekentsprechender Apparates) entfernen.

Die im Haushalt, namentlich für Waschzweeke (Gelbwerden der Wäsche, Erzeugung von Rostflecken), recht störenden Eisenverbindun$g c n^{5}$ ) im Wasser lassen sich durch kleine geeignete Hausapparate ( $z$. B. Dunbarsehes Faß) gut entfernen.

Reinigung des Wassers für technische Zwecke. Wässer, die für technische $Z$ wecke geeignet sein sollen, müssen in der Regel klar, möglichst farbund geruchlos, von nicht saurer Reaktion, praktisch eisen- und manganfrei und vor allen Dingen weich sein. Auch viel Stickstoffverbindungen, organische Stoffe, Chloride und Sulfate im Wasser stören mehr oder weniger ${ }^{3}$ ).

1) H. Klut, Die Bedeutung der freien Kohlensäure im Wasserversorgungswesen. Gesundheits-Ingenieur 1907 , Nr. 32, S. 517 , Bd. 30.

2) H. Wehner, Bleilösung und Eisenlösung bei Wasser versorgungswässern und die Vakuumrieselung, Gesundheit 1908, S. 747 .

3) Handbuch der Hygiene (1. c.), S. 93, Bd. Wasser und Abwasser.

4) Dale, Gesundheits-Ingenieur 1910, Bd. 33, S. 457, u. Schwarz u. Aumann, ebenda 1911, Bd. 34, S. 654.

5) K. Opitz, Klinisoles Jahrbuoh 1912. Bd. 26 . S. 449 .

b) Vergl, a. II. Stoof, Wasser in ,Chemische Technologie der Neuzeit" von 0. Dammer, Bd. 1. Stuttgart 1910. 
Man bezeichnet im allgemeinen Wässer mit einer Gesamthärte (deutsche Grade)

$$
\begin{aligned}
& \text { von } 0-4^{0} \text { sehr weich, } \\
& , " 5-8^{\circ} \text { weich, } \\
& , \quad 9-12^{0} \text { mittelhart, } \\
& \text {, } 13-18^{\circ} \text { ziemlich hart, } \\
& \text { ü } 19-30^{\circ} \text { hart, } \\
& \text { über } 30^{\circ} \text { sehr hart. }
\end{aligned}
$$

Hartes Wasser verbraucht bekanntlich zum Waschen viel Seife und zwar vernichten 20 Härtegrade $2,4 \mathrm{~kg}$ Seife im cbm Wasser. Auch für Kochzwecke ist solches Wasser nur wenig geeignet. Man weiß, daB z. B. Hülsenfrüchte in hartem Wasser schwer weich kochen, und die Herstellung von verschiedenen Getränken, wie Kaffee, Tee usw., erschwert wird.

Eine hervorragende Bedeutung lat die Lutbärtung des Wassers besonders für Kesselspeiseawecke $\left.{ }^{1}\right)$. Harte Wässer sind infolge Steinbildung suhädlich. Die Harte eines Wassers, die bekanntJich durch Kalk- und Magnesiaverbindungen belingt wird, sollte am besten für Kesselspeisezwecke unter $6^{0}$ (deutsche Grade) betragen. 1 deutscher II ärtegrad ist gleich $10 \mathrm{mg} \mathrm{CaO}$ (Kalk) oder 7,1. mg MgO (Magnesia) in 11 Wasser. - Für dic Enthärtung sind heute hauptsächlich drei Verfahren in Gebratch: 1. das Kalk-Sodaverfahren. 2. das Reisertseho Baryberfahren und 3. tas Ganssche Permulituorfahren. Dio Reinigung des Wassers geschieht am besten auberhalb des Damptkicssels.

Das Kalk-Sodaverfahren ist das alteste, ist billig und hat sich in der Praxis im allgemeinen gut bewährt. Zweckmäßig wird die Reinigung in bresonderen Apparaten mit dem vorgewärmten Wasser vorgenommen, weil hierdurth die Enthärtung des Wassers vollkommener ist.

Das Reisertsche Barytuerfahren empfiehlt sich numentich bei hoher Gipshärte des Wassers. Der ziemlich hohe Preis sowie die Giftigkeit der Barytverbindungen sind aber bei dieser Art der Reinigung von Nachteil.

Das jüngste in der Praxis angewandte Reinjgungsverfahren ist die Behandlung des Wassers mit Permutit naeh $R$. Gans $s^{2}$, es wird bereits vielfach mit Erfolg benutzt. Das Permutit ist eine komplizierte, zusammengesetzte, chemische Verbindnng aus Natrinm- und Aluminiumverbindungen mit Kieselsäure. In der Natur kommen diese Stoffe vor als Zeolithe. Permutit ist der Name für das künstich durch Schmelzen von. Aluminiumsilikaten mit Soda hergestellte Produkt. Die Permutite haben die merkwürdige Eigenschatt, daB, wenn man ein kalk-, magnesia-, manganoder eisenhaltiges Wasser durch sie hindurchfiltricrt, sie diese Basen durch Umtausch aufnehmen und dafür eine äquivalente Menge Natrium an das Wasser abgeben. Zur Regeneration der Filter dient Kochsalzlösung. Im Gegensatz zu.

1) Vergl, a. H. Heidepriem, Die Reinigung des Kesselspeisewassers. 2. Aufl. Berlin 1909 .

2) Vergl. Wasser u. Abwasser, Bd. 1, 1909, S. 493 1.. Bil. 5,1912 , S. 113. den beiden anderen Enthärtungsverfahren, welche beide gleichmäßig im allgemeinen nur bis auf etwa 3 bis 4 deutsche Grade enthärten können, erreicht man beim Permutitverfahren bei sorgfältigem Betriebe die Enthärtung des Wassers bis auf 0 Grade. Ein weiterer Vorzug liegt ferner darin, daß hier jegliche Dosierung wegfällt. Bei Wässern mit schwankender chemischer Zusammensetzung, ist das Permutit deshalb besonders geeignet. Enthält das zu reinigende Wasser viel Karbonate des Caleiums und Magnesiums, so enthält das enthärtete Wasser eine entsprechende Menge von Soda. Da nun ein gröBerer Uberschuß von Soda im Dampfkessel störend ist (z. B. kann hierdureh "Spucken" der Lokomotiven loicht hervorgerufen werden), so ist zur Vermeidung dieses Utbelstandes ein häufiges Abb)

Uber die sonstigen Anforderungen, die man an rin Wasser zum Kesselspeisen zu stellen hat, sei auch folgende Literatur noch emptohlen: H. Bunte und $P$. Filner, Kesselspeisewasser und dessen Reinigung in (t. Eckermann, Berichte über KesselsteinGeheimmittel, Hamburg 1905 und $L$. E. Andés, Der Kesselstein, seine Entstehung und Vorhiitung, Wien und T.eipzig 1010.

\section{Einfluß des Eises auf die Ausgestaltung der Hochgebirgstäler.}

Von Dr. A. Mahlke, Hamburg.

Im Anschlub an einen Vortrag Distels uber die Entstehung der Hochgebirgstäler auf dem Geographentag zu Innsbruck Ende Mai 1912 hat sich eine von mehreren Seiten eifrig betriebene Diskussion entwickelt. Da bei der Bildung der Täler trei Faktoren, Eis, Wasser und Verwitterung mitgewirkt haben, so ist es schwierig, die Wirkung jedes einzelnen dieser luktoren genau zu bestimmen und über die Grenzen dieser Wirkungen müssen leicht Meinungsverschiedenheiten entstehen. So wirl \%. B. angenommen, duB die im allgemeinen $\mathrm{U}$-förmige Gestalt der Titer durch $A$ usmuldung seitans des Eises

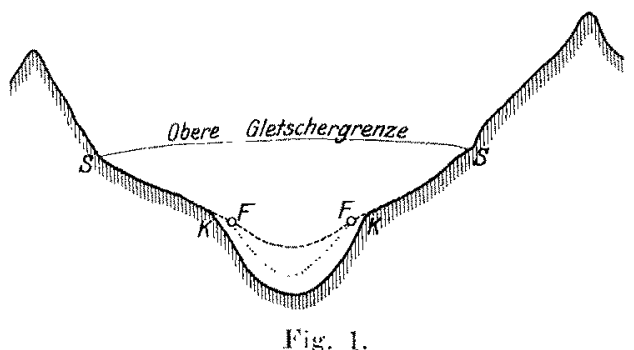

aus $V$-förmigen Tülern hervorgegangen sei. Von anderex Seite wird dagegen behauptet, daß der Talgrund der V-Tiler durch Schntt ausgefullt und so abgerundet sei. 1)ie Talformen sind aber in der Regel noch komplizierter, wie Fig. 1 zeigt. Besonders auffallend sind die einspringende Kante s der Schliffkehle und die vorspringende Kante $K$ des Taltroges, der die Bodenrime des Tales bildet. Penck führt das Einsetzen der starken Erosion bei $S$ auf die Lockerung des Gesteinsgefüges am Gletschersaum zurïck, den Trog aber auf eine maximale Steigerung der Erosionskraft des eiszeitlichen Eisstromes am Orte der gröBten Eismächtigkeit. Er weist daraut hin, daß der Taltrog der Alpen juinger ist, als der 